\title{
New mouse xenograft model modulated by tumor-associated fibroblasts for human multi-drug resistance in cancer
}

\author{
YAN MA $^{2 *}$, ZHIQIANG LIN ${ }^{3 *}$, JOHN K. FALLON $^{1}$, QIANG ZHAO $^{4}$, DAN LIU $^{5}$, \\ YONGJUN WANG ${ }^{6}$ and FENG LIU ${ }^{1 \dagger}$ \\ ${ }^{1}$ Division of Molecular Pharmaceutics, Eshelman School of Pharmacy, University of North Carolina, Chapel Hill, NC, USA; \\ ${ }^{2}$ School of Chinese Materia Medica, Guangzhou University of Chinese Medicine, Guangzhou, Guangdong 510006; \\ ${ }^{3}$ Department of Pharmaceutics, School of Pharmaceutical Sciences, Peking University, Beijing 100191; ${ }^{4}$ College of \\ Chemical Engineering, Sichuan University, Chengdu, Sichuan 610065; ${ }^{5}$ Key Laboratory of Structure-Based Drug Design \\ and Discovery (Shenyang Pharmaceutical University), Ministry of Education, Shenyang, Liaoning 110016; \\ ${ }^{6}$ School of Pharmacy, Shenyang Pharmaceutical University, Shenyang 110016, P.R. China
}

Received June 24, 2015; Accepted July 27, 2015

DOI: 10.3892/or.2015.4265

\begin{abstract}
We developed an MDR tumor model that is modulated by tumor-associated fibroblasts. Studies on proliferation of tumor cell lines including paclitaxel-sensitive and resistant cell lines were performed. The expressions of P-gp and $\alpha$-smooth muscle actin ( $\alpha$-SMA) antigen were evaluated by immunohistochemistry and western blot analysis. Quantitative P-gp analyses of different cell lines were accomplished by nanoUPLC-MS/MS. Tumor cell colony formation assay and established xenograft model was used to investigate the relationship between $\mathrm{P}$-gp expression, fibroblast levels and tumorigenesis. The mouse xenograft model was developed after co-inoculation with MDR tumor cells and NIH/3T3 fibroblast cells. There was no correlation between tumorigenesis in vivo and the growth rate of cells in vitro. The proliferation among different cell lines had no significant differences, but the P-gp expression and tumor growth in the xenograft model were fairly different. P-gp determination and $\alpha$-SMA immunofluorescence staining clarified the relationship between P-gp expression, fibroblast levels and tumorigenesis. It was more difficult for tumor cells with higher P-gp levels to recruit fibroblasts in vivo, resulting in lower tumorigenesis due to the lack of structural
\end{abstract}

Correspondence to: Dr Yan Ma, School of Chinese Materia Medica, Guangzhou University of Chinese Medicine, Guangzhou, Guangdong 510006, P.R. China

E-mail: mygzhtcm@163.com

*Contributed equally

†In memoriam

Key words: xenograft model, fibroblast, tumorigenesis, multi-drug resistance, $\mathrm{P}$-gp expression and chemical support during tumor progression. In the established paclitaxel-resistant mouse xenograft model, no obvious antitumor effect was observed after Taxol treatment, but a significant decrease in tumor size for the group treated with gemcitabine sensitive to the model. The results show that the added fibroblasts do not disturb the applicability of the model in MDR. Therefore, this mouse xenograft MDR model could serve as an effective tool for MDR research.

\section{Introduction}

A major obstacle to successful tumor chemotherapy is multidrug resistance (MDR), which has been widely investigated in recent decades (1). Numerous studies have been conducted into MDR mechanisms and strategies for overcoming MDR. Unfortunately, most patients still die from their disseminated cancer due to resistance to available anticancer drugs (2). Therefore, the development of an MDR model to screen novel therapy regimens for translation from the laboratory to the clinic is desperately needed.

An important tool in MDR research, the tumor models include three main types: the planar cell model, the threedimensional tumor model in vitro and the animal xenograft model. The in vitro monolayer cell model is easy to establish and is thus widely used in the early phase of tumor research. Its value, however, is limited due to its lack of relevance to clinical samples (3). Moreover, apart from the MDR tumor cell itself, the tumor microenvironment also plays an important, even dominant, role in MDR (4). The interaction between cells and extracellular matrices is extremely complex (5). To better mimic tumors in vivo, the three-dimensional matrix model was developed, leading to more physiologically relevant conditions for assessing drug responses and studying biological mechanisms. In vitro $2-\mathrm{D}$ cell and $3-\mathrm{D}$ matrix models play an important role in understanding complex tumor systems but both are hardly sufficient for evaluating the real effects of products on tumors in vivo (6). Therefore, a whole animal xenograft model needs to be developed. 
When developing tumor models an issue with which many groups are confronted is that it is difficult for certain types of MDR tumor cell lines to grow with appropriate rate in vivo after being inoculated subcutaneously, even though the cells may grow quickly in a planar culture dish. In our laboratory, we found an interesting scientific phenomenon that three resistant tumor cell lines of KB-8-5, H460/Tax-R and NCI/ADR-RES with gradually increasing expression of $\mathrm{P}$-gp have similar proliferation rate in vitro, but in the xenograft model the higher the P-gp was expressed, the harder the model was to be establish. Normally, the xenograft models of KB-8-5 and H460/ Tax-R carcinoma lines in nude mice can be established within 5 and 12 days or so, while the tumor of NCI-ADR-RES cell lines in the nude mouse model shows hardly any growth (data not shown). In addition, the resistant KB-8-5 tumor models take on satisfactory reproduction and stability. Some tumor cell lines with low tumorigenesis have been reported (7), but few studies focus on the tumorigenic ability of MDR cells that have high P-gp (also known as MDR1; multidrug resistance protein 1) expression. Thus, we undertook such a study and developed a fast growing whole animal MDR tumor model with high level of P-gp expression.

Aside from the biological characteristics of tumor cells themselves, the tumor microenvironment contributes greatly to tumor growth and drug resistance (8). Fibroblasts, the most numerous and most important cells found in the tumor microenvironment, could affect tumor cell morphology, adhesion, proliferation and signaling by both structural and chemical means $(6,9)$. Tumor-associated fibroblast (TAF) cells have been found to play a critical role in regulating the growth of adjacent tumor cells and vein endothelial cells via different growth factors such as VEGF, HGF and bFGF. TAF cells promote tumorigenesis, and tumor cells that fail to recruit fibroblasts develop slowly $(9,10)$. Some groups have employed fibroblasts to accelerate the growth rate of tumors by co-inoculation with cancer cell lines MCF-Ras, human PC-3 and MDA-436 (7,11). Solid tumor growth depends greatly on the formation of the stroma. Without mesenchyme, a tumor $<2 \mathrm{~mm}$ in length cannot continue to grow (12).

Previous studies in our laboratory had already found highly significant expression of TAF cells within the harvested KB-8-5 tumor through the immunofluorescence of $\alpha$-smooth muscle actin ( $\alpha$-SMA) in the tumor parafin sections and paclitaxel (Taxol) had no effect on the inhibition of tumor growth (unpublished data). Consequently, we hypothesize the in vivo growth of resistant tumor cells with high P-gp expression in nude mice depends on the TAF cells to a certain extent. We investigated the correlations among tumorgenesis, tumor cell growth profile in vitro, $\mathrm{P}-\mathrm{gp}$ expression levels and fibroblast distribution profiles in tumor sections, and then develop a new in vivo MDR tumor mouse model by co-inoculating with resistant tumor cells and $\mathrm{NIH} / 3 \mathrm{~T} 3$ fibroblasts, which may be useful for the screening of antineoplastic agents and mechanistic studies of factors regulating tumor growth and progression.

\section{Materials and methods}

Materials. Taxol injection was manufactured by Hospira, Inc. (Lake Forest, IL, USA). Gemcitabine was purchased from
Sigma-Aldrich (St. Louis, MO, USA). Antibodies against P-gp, $\alpha$-SMA and GAPDH, horseradish peroxidase-conjugated anti-mouse and anti-rabbit whole $\mathrm{IgG}$ for western blot and fluorescence-conjugated anti-mouse and anti-rabbit whole IgG for immunofluorescence assay, were obtained from Santa Cruz Biotechnology (San Diego, CA, USA). Matrigel ${ }^{\mathrm{TM}}$ Basement Membrane Matrix was purchased from BD Biosciences (San Jose, CA, USA).

Sensitive KB-3-1 cell line (human mouth epidermal carcinoma cells) and the P-gp-overexpressing NCI/ADR-RES cell line (human ovarian carcinoma cells) were obtained from the National Cancer Institute (Bethesda, MD, USA). Resistant KB-8-5 cells were separated from KB-3-1 cells. H460/Tax-R cell line (non-small lung carcinoma cells) was obtained from Dr Bingliang Fang at the MD Anderson Cancer Center (Houston, TX, USA). NIH/3T3 fibroblast cells (mouse embryonic fibroblast cell line) were originally obtained from the American Type Culture Collection (ATCC; Manassas, VA, USA). Cells were maintained in RPMI-1640 or Dulbecco's modified Eagle's medium (DMEM) containing 10\% fetal bovine serum (FBS), $100 \mathrm{U} / \mathrm{ml}$ penicillin and $100 \mu \mathrm{g} / \mathrm{ml}$ streptomycin (all from Invitrogen, Carlsbad, CA, USA). Cells were cultivated at $37^{\circ} \mathrm{C}$ with $5 \% \mathrm{CO}_{2}$ in a humidified incubator.

Nude mice were purchased from the National Cancer Institute. All experiments performed on animals were in accordance with and approved by the Institutional Animal Care and Use Committee at the University of North Carolina at Chapel Hill.

Western blot analysis. For in vitro preparations adhered cells in culture dishes were washed with ice-cold PBS, scraped off the dish using a cold plastic cell scraper, and the suspension gently transferred to a pre-cooled Eppendorf tube. For in vitro studies the tumor-bearing mice were sacrificed and the tumors were removed. Whole cell suspensions or tumor lysates were extracted with RIPA buffer $(\sim 10 \mathrm{mg}$ of tumor was mixed with $100 \mu \mathrm{l}$ RIPA buffer), and the concentration of protein was quantified using a Pierce BCA Protein Assay kit (Thermo Scientific Inc., Rockford, IL, USA). Approximately $50 \mu \mathrm{g}$ of protein from each sample was separated on a NuGAGE $12 \%$ SDS-polyacrylamine gel, and then transferred to a polyvinylidene difluoride (PVDF) membrane (Bio-Rad, Hercules, CA, USA). The membrane was blocked with 5\% skimmed milk in PBS for $1 \mathrm{~h}$. After incubation with primary antibody at $4^{\circ} \mathrm{C}$ overnight, the PVDF membrane was washed with PBST (0.2\% Tween-80 in PBS), and then incubated with secondary antibody for $1 \mathrm{~h}$ at room temperature. Antibodies against P-gp, $\alpha$-SMA and GAPDH were used at 1:2,000 dilutions. A HRP-conjugated anti-mouse antibody at a dilution of 1:10,000 or an HRP-conjugated anti-rabbit IgG at a dilution of 1:2,000 served as the secondary antibodies in the experiment. The specific protein bands were visualized using a chemiluminescence kit (Pierce, Rockford, IL, USA). Chemiluminiscent signals were detected with the high-performance chemiluminescence film (GE Healthcare Bio-Sciences, Pittsburgh, PA, USA).

Targeted quantitative proteomic analysis for P-gp determination. Samples were prepared by modification of previously published targeted quantitative methods of ours $(13,14)$. 
Briefly, cells ( $\sim 10$ million), to which $0.5 \mathrm{ml} 50 \mathrm{mM}$ ammonium bicarbonate had been added, were homogenized in a 2-ml glass homogenizer (Wheaton, Millville, NJ, USA). Following centrifugation at $16,000 \mathrm{x}$ g for $10 \mathrm{~min}$ the supernatant was discarded and the pellet solubilized, with further homogenization, in $0.5 \mathrm{ml}$ of $1 \%$ sodium deoxycholate. The homogenate was centrifuged again at $16,000 \mathrm{x}$ g for $10 \mathrm{~min}$ and the pellet discarded. Total protein concentration of the supernatant was measured using the Pierce BCA Protein Assay kit (Thermo Scientific Inc.).

Membrane samples (25 $\mu \mathrm{g} /$ replicate) were analyzed in duplicate. $\beta$-casein $(0.5 \mu \mathrm{g}$, from bovine milk; SigmaAldrich) was added as a general indicator of successful digestion. Samples were denatured with heat, reduced with dithiothreitol and carbamidomethylated with iodoacetamide. Trypsin solution $(0.01 \mu \mathrm{g} / \mu \mathrm{l})$ was added to give a cell protein to trypsin ratio of $20: 1$ and samples were digested at $37^{\circ} \mathrm{C}$ for $4 \mathrm{~h}$ ( $\mathrm{pH} \sim 8 ; 50 \mathrm{mM}$ ammonium bicarbonate buffer), shaking at $300 \mathrm{rpm}$. The reaction was stopped by addition of $10 \%$ trifluoroacetic acid, the amount added being $10 \%$ of the total reaction volume. Following centrifugation at $13,400 \times \mathrm{g}$ for 5 min the supernatant was treated with solid phase extraction (C18 cartridges, Strata-X, 33u, polymeric, $10 \mathrm{mg} / \mathrm{ml}$; Phenomenex, Torrance, CA, USA). The eluate was evaporated, reconstituted and analyzed by nanoUPLC-MS/MS as previously described $(13,14)$.

Two of the proteotypic P-gp tryptic peptides targeted in the nano-UPLC-MS/MS analysis were $\mathrm{I}_{368}$ IDNKPSIDSYSK $_{380}$ and $\mathrm{I}_{896} \mathrm{ATEAIENFR}_{905}$. Two MRMs were acquired per peptide and peak areas were used to compare samples. The two MRM areas were averaged and then the areas for the duplicates were averaged. Results for peptides were compared between cell samples.

Cell proliferation assay. Four types of tumor cell lines, namely KB-3-1, KB-8-5, H460/Tax-R and NCI/ADR-RES cells, were seeded in 6-well plates at a density of 40,000 cells/well and incubated at atmospheric pressure at $37^{\circ} \mathrm{C}$ with $5 \% \mathrm{CO}_{2}$. Over the following week, cells in each well were harvested and counted using a cell counting plate. The cell number was plotted against time.

Tumor cell colony formation assay. To perform the tumor cell colony formation assay, 1.2 and $0.7 \%$ agarose solutions were first prepared. The solutions were melted in a microwave oven and cooled to $40^{\circ} \mathrm{C}$ in a water bath before use. An equal volume of $1.2 \%$ agarose was mixed with 2x RPMI-1640 containing $20 \% \mathrm{FBS}$ and antibiotics to give base agarose. One milliliter of this mixture was added to each well of a 6-well plate and allowed to solidify for $5 \mathrm{~min}$. Following this, $1 \mathrm{ml}$ of $0.7 \%$ agarose, mixed with $2 \mathrm{x}$ RPMI-1640 and 2,500 cells, was plated gently onto the surface of the base agarose. 1x RPMI-1640 (0.5 ml) of medium was then added to each well and the plate was incubated at $37^{\circ} \mathrm{C}$ for 15 days. Cells were washed 1-2 times/week with cell culture medium. At day 15, cell colonies were photographed after staining with $0.5 \mathrm{ml}$ of $0.005 \%$ crystal violet for $15 \mathrm{~min}$.

Mouse xenograft model development and tumor growth. Female nude mice (6-8 weeks) were used in all studies. The mice were divided into 6 groups, each receiving the following cells via subcutaneous injection into their right or left flanks: i), KB-3-1 cells $\left(5 \times 10^{6}\right)$; ii), KB-8-5 cells $\left(5 \times 10^{6}\right)$; iii), H460/Tax-R cells $\left(5 \times 10^{6}\right)$; iv), NCI/ADR-RES cells $\left(5 \times 10^{6}\right)$; v), H460/Tax-R $\left(5 \times 10^{6}\right)$ and NIH/3T3 cells $\left(2.5 \times 10^{6}\right)$; and vi), NCI/ADR-RES $\left(5 \times 10^{6}\right)$ and NIH/3T3 cells $\left(2.5 \times 10^{6}\right)$. Cells of group $v$ and vi were mixed with Matrigel ${ }^{\mathrm{TM}}$ Basement Membrane Matrix at a ratio of 2:1 (w/w) before injection. Images of the mice were taken with a digital camera 16 days after tumor cell inoculation.

Sensitivity of the MDR tumor model to chemotherapy. Tumor sensitivity to chemotherapy was evaluated in the NCI/ADR-RES and H460/Tax-R cell-bearing nude mouse models. Once the tumor mass in the xenograft was established, mice were randomly divided into 3 groups ( 5 mice/group) and were injected, in the tail vein, with normal saline (the control group), Taxol or gemcitabine. Drug doses of $10 \mathrm{mg} / \mathrm{kg}$ PTX and $9 \mathrm{mg} / \mathrm{kg}$ gemcitabine were used for all treatments. Therapy was continued at days 3,5, 7 and 9 (5 doses in total). Mice were sacrificed when the tumor reached $2 \mathrm{~cm}$ in length, and tumor volumes were calculated using the following equation: (length $\mathrm{x}$ width $\left.^{2}\right) / 2$.

Immunofluorescence assay. Harvested tumors were fixed in $4.0 \%$ paraformaldehyde (PFA), paraffin-embedded, and sectioned at the UNC Lineberger Comprehensive Cancer Center Animal Histopathology Facility. For immunofluorescence of P-gp and $\alpha$-SMA, slides were subjected to block by BSA after deparaffinization, dehydration and antigen retrieval. Slides were then incubated with primary antibodies (diluted by $1 \% \mathrm{BSA}$ ) at $4^{\circ} \mathrm{C}$ overnight and with secondary antibodies at room temperature for $1 \mathrm{~h}$. Nuclei were fluorescently stained with 4',6-diamidino-2-phenylindole (DAPI) Vectashield (Vector Laboratories, Inc., Burlingame, CA, USA). Finally, all slides were photographed at 20 -fold magnification. The primary antibodies for P-gp and $\alpha$-SMA were diluted 200 -fold and the secondary antibodies 100 -fold.

\section{Results and Discussion}

Tumorigenesis of various tumor cell lines in vivo. KB-3-1, KB-8-5, H460/Tax-R and NCI/ADR-RES are the four frequently used cell lines in tumor research. However, their tumor growth characteristics in vivo vary greatly. As shown in Fig. 1, obvious differences in tumorigenesis were observed in nude mice received subcutaneous inoculation with one of the four types of cell line. The graph in Fig. 1 shows that KB-3-1 and KB-8-5 grew much faster than H460/Tax-R and NCI/ADR-RES in vivo. The tumor volume increment of KB-8-5 was about 5- and 56-fold compared with that of H460/Tax-R and NCI/ADR-RES. It suggests that KB-3-1 and $\mathrm{KB}-8-5$ are the most tumorigenic of the cells. Only a 'tumor dot' was observed in the mouse that was injected with H460/ Tax-R cells and no tumor was observed in the NCI/ADR-RESbearing mouse.

Correlations among cell proliferation, colony formation, $P$-gp expression and tumorigenesis in vitro and in vivo. To explore the reasons why tumorigenesis differs among tumor cell types, we firstly compared the growth rate and colony 


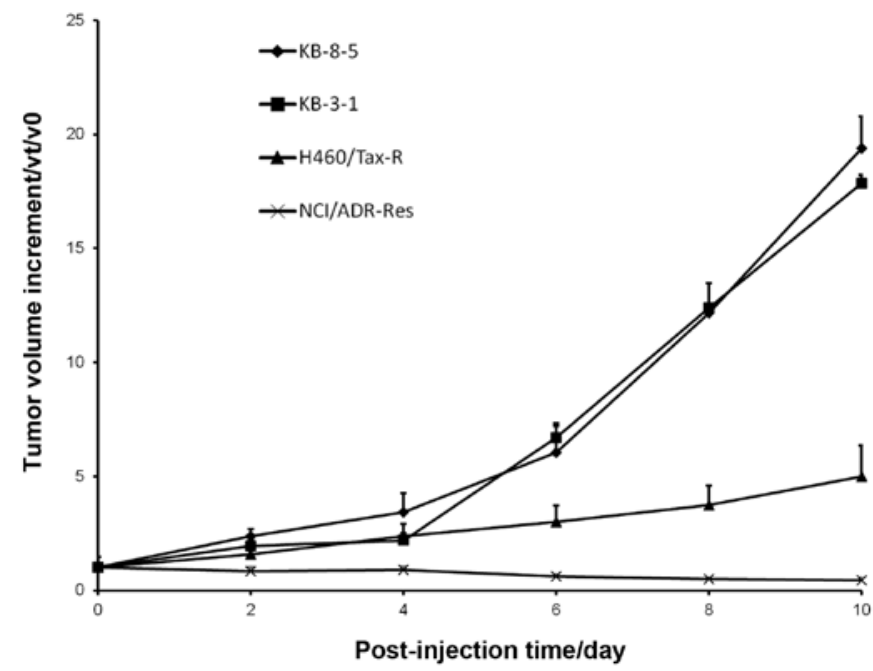

Figure 1. Tumor growth rate in nude mice administered subcutaneous inoculation with one of the four types of cell line.

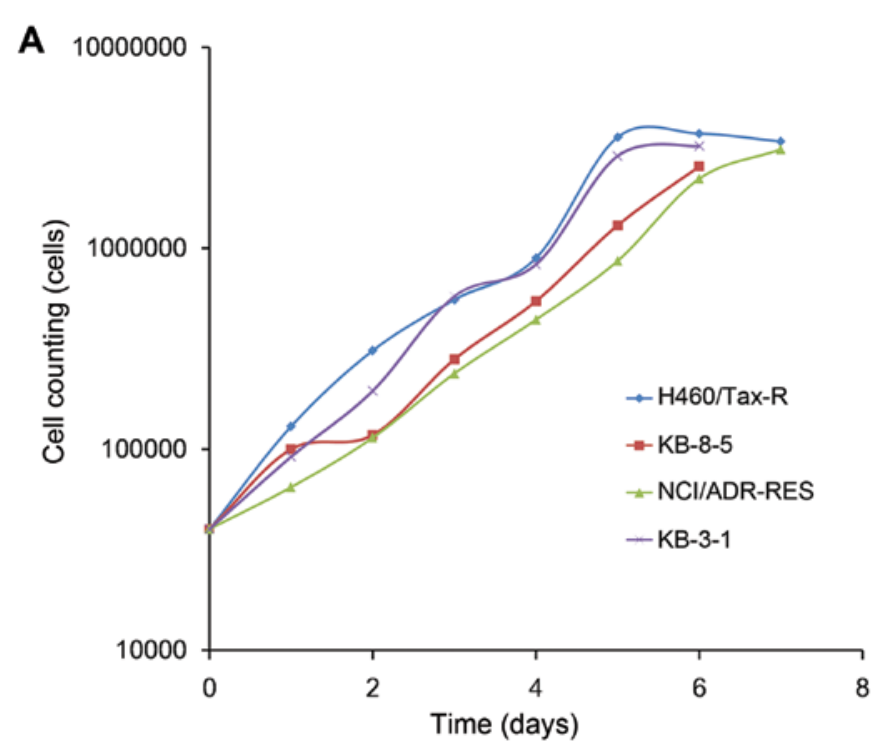

B

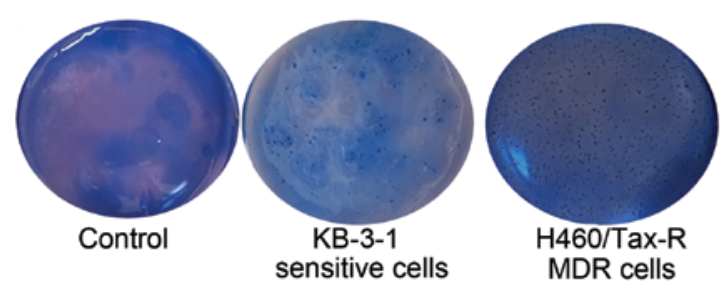

Figure 2. Tumor cell growth profiles in vitro. (A) Cell growth curves of various tumor cell lines. (B) Colony formation of drug sensitive cells (KB-3-1) and MDR cells (H460/Tax-R). MDR, multidrug resistance.

formation ability of the cell lines in vitro. As shown in Fig. 2A, comparable rates of tumor cell proliferation was observed in plates regardless of their cell type and sensitivity. All of the tumor cells grew quickly in vitro and maintained proliferation activity.

The colony formation assay is an in vitro cell survival assay based on the ability of a single cell to grow into a colony, reflecting the possible malignant properties of tumor cells. Correlations between tumor cell colony formation and tumor
A
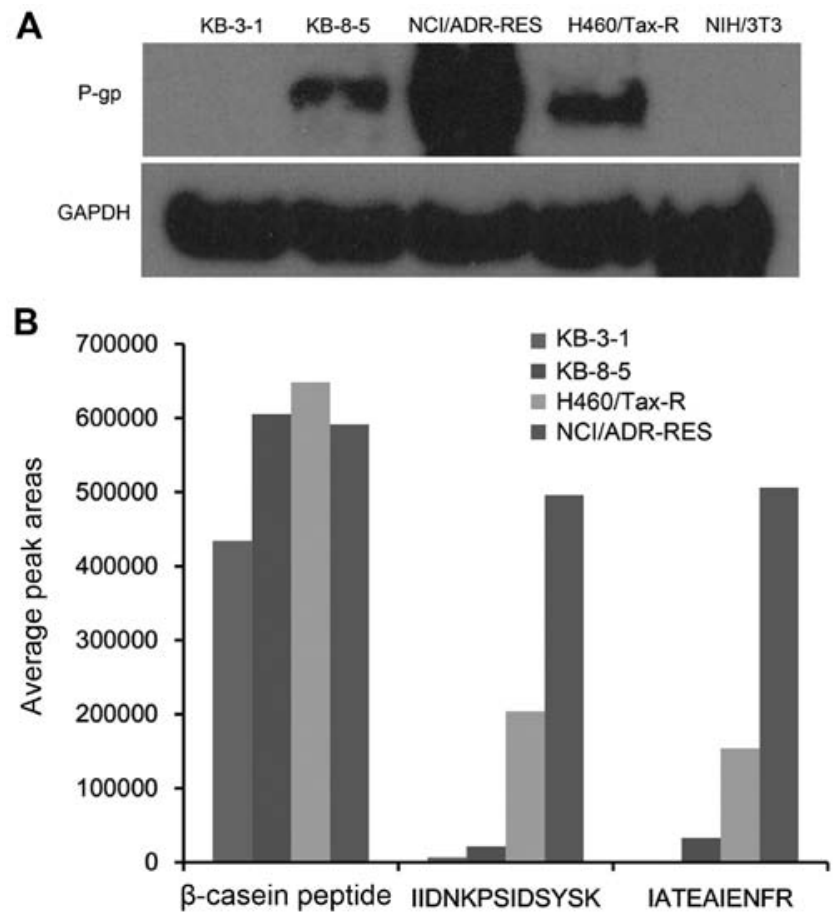

Figure 3. P-gp expression in various cells determined by western blot (A) and targeted quantitative proteomic analysis (B).

growth in vivo have previously been reported $(15,16)$. In our comparisons, both of drug sensitive KB-3-1 cells and the drug resistant H460/Tax-R cells preferred colony formation (Fig. 2), but these two types of cells presented completely different growth rate in the xenograft mouse (Fig. 1). Hitherto, there existed no correlation between colony formation and tumor growth in vivo.

In the mouse xenograft models we found the growth of drug resistant tumors (e.g., NCI/ADR-RES and H460/Tax-R) to be slow (Fig. 1). In order to characterize the drug resistance we measured P-gp expression in different cell lines qualitatively using western blot analysis and nanoUPLC-MS/MS. As shown in Fig. 3, both methods showed the same trend of P-gp expression in the cell lines: NCI/ADR-RES > H460/Tax-R > KB-8-5 $>$ KB-3-1. Slight P-gp expression was seen in KB-3-1 cells, which are sensitive to Taxol (17). More P-gp expression was observed in the other three resistant cell types (17). It followed that cells containing high P-gp levels (Fig. 3) showed low tumorigenesis in vivo (Fig. 1). It was therefore suggested that NIH/3T3 fibroblasts without P-gp expression (Fig. 3) would be tested as auxiliary cells for development of a resistant in vivo tumor model.

Analysis of tissue section of the low tumorigenesis tumor (H460/Tax-R). To further study the apparent negative correlation between high P-gp levels and tumorigenesis, we assayed H460/Tax-R tumor tissue sections by immunofluorescence staining. Staining for $\alpha$-SMA was performed because its expression is the major morphological characteristic of myofibroblasts which contribute greatly to tumor stroma and tumor growth in vivo (18). As shown in Fig. 4, abundant TAFs in the fast growing KB-8-5 tumor, and few TAFs in the H460/Tax-R tumor were observed, which had been a 'tumor dot' in the 


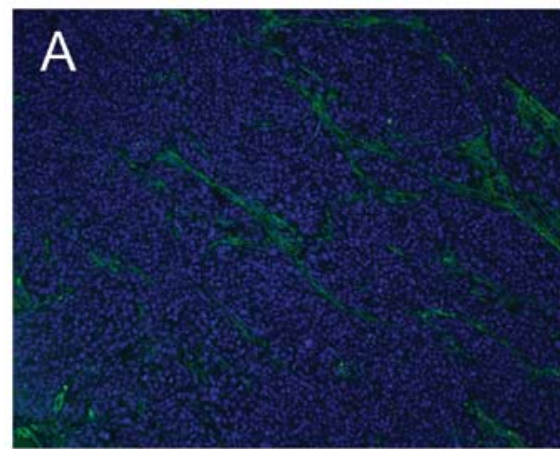

KB-8-5

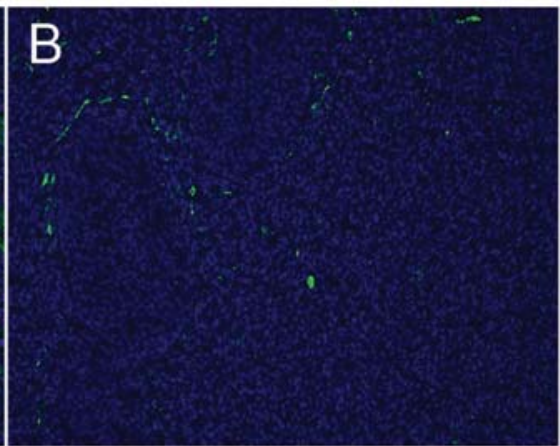

H460/Tax-R

Figure 4. Immunofluorescence staining for $\alpha$-SMA expression in KB-8-5 (A) and H460/Tax-R (B). Nude mice were inoculated with KB-8-5 or H460/Tax-R cells subcutanously and sacrificed after 16 days. Tumor tissues were harvested and sectioned for immunofluorescence analysis following a common protocol. $\alpha$-SMA, $\alpha$-smooth muscle actin.
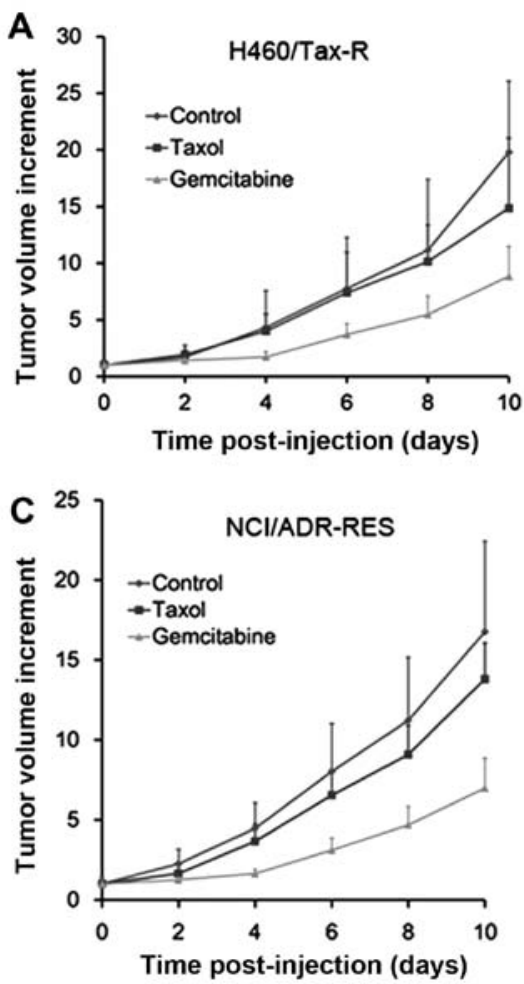

B

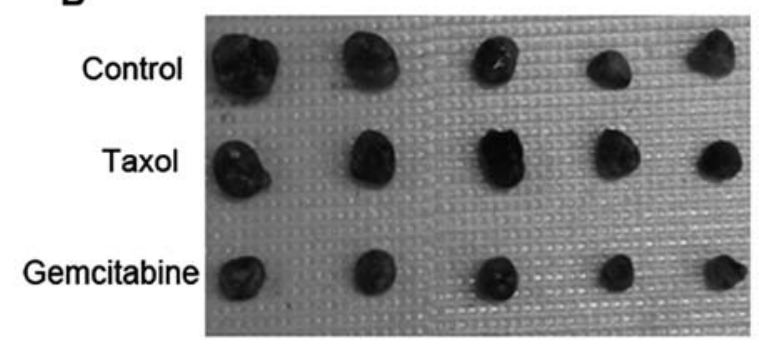

D

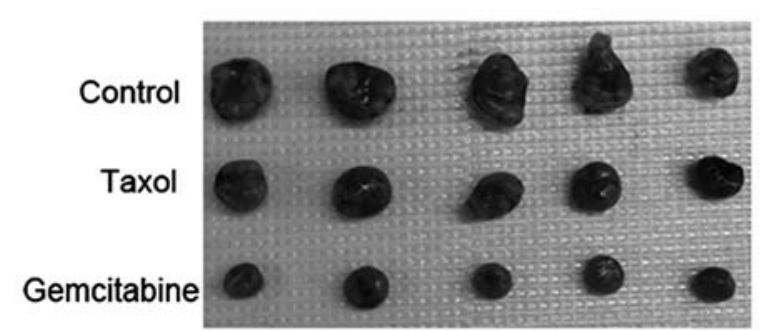

Figure 5. Sensitivity of the new mouse xenograft model to chemotherapy drugs. (A) H460/Tax-R tumor size progression as a function of time post-administration. (B) Photographs of excised H460/Tax-R tumors after receiving different treatments. (C) NCI/ADR-RES tumor size progression as a function of time post-administration. (D) Photographs of excised NCI/ADR-RES tumors after receiving different treatments.

mouse xenograft model (Fig. 1). The strong effect of TAFs on tumor growth has been widely reported $(7,19)$. We believe that tumor cells in vivo containing high levels of P-gp have difficulty in recruiting TAFs, resulting in a lack of structural and chemical support necessary for tumor progression (20). We therefore decided to develop and test an MDR xenograft mouse model produced by co-inoculation with tumor cells and fibroblast cells.

Establishment and evaluation of a new mouse xenograft model. As expected, the MDR tumor model was successfully produced by co-inoculation with NIH/3T3 fibroblast (TAF) cells and H460/Tax-R or NCI/ADR-RES cells (Fig. 5B and D).
In our preliminary experiments, different ratios of tumor cells to $\mathrm{NIH} / 3 \mathrm{~T} 3$ cells $(2: 1$ or $1: 1)$ were tested but no obvious differences in tumor growth were observed. Moreover, the NIH/3T3 cells did not form a tumor after being injected subcutaneously. All the results suggested that improvement of tumorigenesis is mainly due to modulation of the tumor by the fibroblasts and not growth of the fibroblasts themselves.

Tumor sensitivity to chemotherapy was investigated in the developed H460/Tax-R and NCI/ADR-RES models. Taxol, a $\mathrm{P}$-gp substrate, was chosen as the tumor resistant test agent and gemcitabine as the tumor sensitive agent (21-23). As shown in Fig. 5, Taxol exerted little effect on tumor growth for both cell types, indicating the successful establishment of the mouse 


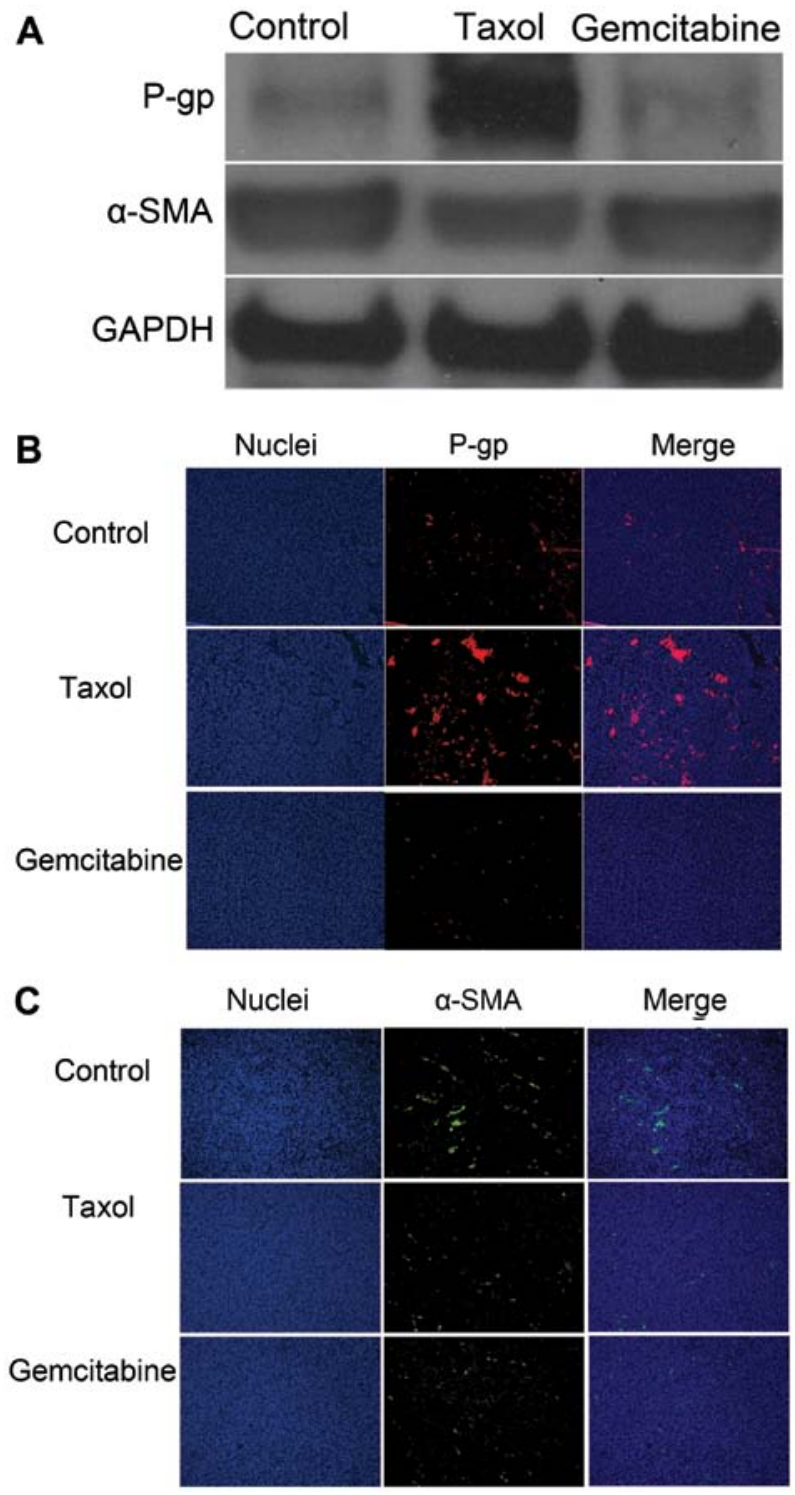

Figure 6. P-gp and $\alpha$-SMA expression in the new NCI/ADR-RES tumor model determined by (A) immunofluorescence and (B and C) western blot analysis. The nuclei were stained with DAPI (blue) and $\alpha$-SMA with Texas Red (red). $\alpha$-SMA, $\alpha$-smooth muscle actin.

xenograft model modulated by TAF cells. The observed anticancer activity of gemcitabine ruled out the possibility that the poor response to Taxol was due to the change in microenvironment of the tumors caused by the TAF cells. It is well known that the weak response to PTX is caused by the efflux of P-gp.

To further evaluate the applicability of the novel MDR tumor model, P-gp and $\alpha$-SMA levels in tumor tissues were determined by western blot and immunofluorescence staining analysis. As shown in Fig. 6B, P-gp expression increased in the MDR tumor after Taxol treatment. This is consistent with previous studies that P-gp expression can be induced by Taxol (24). Higher P-gp levels in the Taxol-treated group confirm that the novel MDR model was successfully developed. In contrast, gemcitabine, to which MDR tumors are sensitive, did not increase P-gp expression in the model (Fig. 6B). The slightly higher level of $\alpha$-SMA expression were observed in the tumors treated with Taxol compared to the control tumors. We supposed that the resistance of Taxol may be related to the increased TAF besides the efflux pump of P-gp. Further research is needed in our subsequent study.
Overall, no antitumor effect was observed after Taxol treatment, mainly because the drug could not kill the MDR tumor cells or the fibroblasts, evident from an increase in P-gp levels and no obvious change in $\alpha$-SMA expression when compared to the untreated group. In contrast, for gemcitabine, a significant decrease in tumor size and little change in $\alpha$-SMA expression suggested that the gemcitabine acted mainly on the tumor cells. Therefore, without being affected by the added fibroblasts, the new human MDR mouse xenograft model should serve as a useful tool to evaluate the antitumor activity of drugs.

In conclusion, in the present study we investigated the common problems of developing a mouse xenograft model using tumor cell lines that highly express P-gp and exhibit low tumorigenesis in vivo.

Failure to recruit fibroblasts in vivo for tumor cells containing high levels of P-gp lies in less structural and chemical support during tumor progression. A new mouse xenograft model applicable to MDR research has been successfully developed by co-inoculating with tumor cells (NCI/ADR-RES 
or H460/Tax-R) and fibroblast cells. The new MDR model is resistant to Taxol treatment, which affects neither the tumor cells nor the fibroblasts. In contrast, gemcitabine, to which MDR tumors are not resistant, inhibits tumor growth by killing tumor cells but exerts no obvious influence on fibroblasts. Fibroblasts in this tumor model therefore would not disturb application of the model in the research of MDR. The new mouse xenograft MDR model should serve as a useful tool to evaluate the antitumor effect of drugs.

\section{Acknowledgements}

This study was supported by the National Cancer Institute Grant (5R01CA149387) and the National Nature Science Foundation of China (no. 81403109) and the Youth Elite Project of Guangzhou University of Chinese Medicine (no. QNYC20140107).

\section{Dedication}

This study was dedicated to the memory of Professor Feng Liu, 1955-2014, University of North Carolina at Chapel Hill.

\section{References}

1. Gottesman MM, Fojo T and Bates SE: Multidrug resistance in cancer: Role of ATP-dependent transporters. Nat Rev Cancer 2: 48-58, 2002.

2. Borst P, Jonkers $\mathrm{J}$ and Rottenberg S: What makes tumors multidrug resistant? Cell Cycle 6: 2782-2787, 2007.

3. Gillet JP, Calcagno AM, Varma S, Marino M, Green LJ, Vora MI, Patel C, Orina JN, Eliseeva TA, Singal V, et al: Redefining the relevance of established cancer cell lines to the study of mechanisms of clinical anti-cancer drug resistance. Proc Natl Acad Sci USA 108: 18708-18713, 2011.

4. Correia AL and Bissell MJ: The tumor microenvironment is a dominant force in multidrug resistance. Drug Resist Updat 15: 39-49, 2012.

5. Grinnell F, Rocha LB, Iucu C, Rhee S and Jiang H: Nested collagen matrices: A new model to study migration of human fibroblast populations in three dimensions. Exp Cell Res 312: $86-94,2006$.

6. Green JA and Yamada KM: Three-dimensional microenvironments modulate fibroblast signaling responses. Adv Drug Deliv Rev 59: 1293-1298, 2007.

7. Orimo A,Gupta PB,Sgroi DC, Arenzana-SeisdedosF,Delaunay T, Naeem R, Carey VJ, Richardson AL and Weinberg RA: Stromal fibroblasts present in invasive human breast carcinomas promote tumor growth and angiogenesis through elevated SDF-1/CXCL12 secretion. Cell 121: 335-348, 2005.

8. Flach EH, Rebecca VW, Herlyn M, Smalley KS and Anderson AR: Fibroblasts contribute to melanoma tumor growth and drug resistance. Mol Pharm 8: 2039-2049, 2011.
9. Cirri P and Chiarugi P: Cancer-associated-fibroblasts and tumour cells: A diabolic liaison driving cancer progression. Cancer Metastasis Rev 31: 195-208, 2012.

10. Dong J, Grunstein J, Tejada M, Peale F, Frantz G, Liang WC, Bai W, Yu L, Kowalski J, Liang X, et al: VEGF-null cells require PDGFR alpha signaling-mediated stromal fibroblast recruitment for tumorigenesis. EMBO J 23: 2800-2810, 2004.

11. Camps JL, Chang SM, Hsu TC, Freeman MR, Hong SJ, Zhau HE, von Eschenbach AC and Chung LW: Fibroblast-mediated acceleration of human epithelial tumor growth in vivo. Proc Natl Acad Sci USA 87: 75-79, 1990.

12. Folkman J: Tumor angiogenesis and tissue factor. Nat Med 2: 167-168, 1996.

13. Fallon JK, Neubert H, Hyland R, Goosen TC and Smith PC: Targeted quantitative proteomics for the analysis of 14 UGT1As and -2Bs in human liver using NanoUPLC-MS/MS with selected reaction monitoring. J Proteome Res 12: 4402-4413, 2013.

14. Higgins JW, Bao JQ, Ke AB, Manro JR, Fallon JK, Smith PC and Zamek-Gliszczynski MJ: Utility of Oatpla/1b-knockout and OATP1B1/3-humanized mice in the study of OATP-mediated pharmacokinetics and tissue distribution: Case studies with pravastatin, atorvastatin, simvastatin, and carboxydichlorofluorescein. Drug Metab Dispos 42: 182-192, 2014.

15. Shin SI, Freedman VH, Risser R and Pollack R: Tumorigenicity of virus-transformed cells in nude mice is correlated specifically with anchorage independent growth in vitro. Proc Natl Acad Sci USA 72: 4435-4439, 1975.

16. Colburn NH, Bruegge WF, Bates JR, Gray RH, Rossen JD, Kelsey WH and Shimada T: Correlation of anchorage-independent growth with tumorigenicity of chemically transformed mouse epidermal cells. Cancer Res 38: 624-634, 1978.

17. Chung HC, Rha SY, Kim JH, Roh JK, Min JS, Lee KS, Kim BS and Lee KB: P-glycoprotein: The intermediate end point of drug response to induction chemotherapy in locally advanced breast cancer. Breast Cancer Res Treat 42: 65-72, 1997.

18. Hinz B, Celetta G, Tomasek JJ, Gabbiani G and Chaponnier C: Alpha-smooth muscle actin expression upregulates fibroblast contractile activity. Mol Biol Cell 12: 2730-2741, 2001.

19. Ostman A and Augsten M: Cancer-associated fibroblasts and tumor growth - bystanders turning into key players. Curr Opin Genet Dev 19: 67-73, 2009.

20. Ishii G, Sangai T, Ito T, Hasebe T, Endoh Y, Sasaki H, Harigaya K and Ochiai A: In vivo and in vitro characterization of human fibroblasts recruited selectively into human cancer stroma. Int J Cancer 117: 212-220, 2005

21. Casazza AM and Fairchild CR: Paclitaxel (Taxol): Mechanisms of resistance. Cancer Treat Res 87: 149-171, 1996.

22. Davidson JD, Ma L, Flagella M, Geeganage S, Gelbert LM and Slapak CA: An increase in the expression of ribonucleotide reductase large subunit 1 is associated with gemcitabine resistance in non-small cell lung cancer cell lines. Cancer Res 64: 3761-3766, 2004.

23. Bergman AM, Pinedo HM, Talianidis I, Veerman G, Loves WJ, van der Wilt CL and Peters GJ: Increased sensitivity to gemcitabine of P-glycoprotein and multidrug resistance-associated protein-overexpressing human cancer cell lines. Br J Cancer 88: 1963-1970, 2003.

24. Yusuf RZ, Duan Z, Lamendola DE, Penson RT and Seiden MV: Paclitaxel resistance: Molecular mechanisms and pharmacologic manipulation. Curr Cancer Drug Targets 3: 1-19, 2003. 